\title{
Front Matter: Volume 7302
}

, "Front Matter: Volume 7302," Proc. SPIE 7302, Window and Dome Technologies and Materials XI, 730201 (26 May 2009); doi:

$10.1117 / 12.833546$

SPIE Event: SPIE Defense, Security, and Sensing, 2009, Orlando, Florida, United SPIE. States 


\section{PROCEEDINGS OF SPIE}

\section{Window and Dome Technologies and Materials XI}

Randal W. Tustison

Editor

15-16 April 2009

Orlando, Florida, United States

Sponsored and Published by

SPIE 
The papers included in this volume were part of the technical conference cited on the cover and title page. Papers were selected and subject to review by the editors and conference program committee. Some conference presentations may not be available for publication. The papers published in these proceedings reflect the work and thoughts of the authors and are published herein as submitted. The publisher is not responsible for the validity of the information or for any outcomes resulting from reliance thereon.

Please use the following format to cite material from this book:

Author(s), "Title of Paper," in Window and Dome Technologies and Materials XI, edited by Randal W. Tustison, Proceedings of SPIE Vol. 7302 (SPIE, Bellingham, WA, 2009) Article CID Number.

ISSN 0277-786X

ISBN 9780819475688

Published by

SPIE

P.O. Box 10, Bellingham, Washington 98227-0010 USA

Telephone +1 3606763290 (Pacific Time) · Fax +1 3606471445

SPIE.org

Copyright (C) 2009, Society of Photo-Optical Instrumentation Engineers

Copying of material in this book for internal or personal use, or for the internal or personal use of specific clients, beyond the fair use provisions granted by the U.S. Copyright Law is authorized by SPIE subject to payment of copying fees. The Transactional Reporting Service base fee for this volume is $\$ 18.00$ per article (or portion thereof), which should be paid directly to the Copyright Clearance Center (CCC), 222 Rosewood Drive, Danvers, MA 01923. Payment may also be made electronically through CCC Online at copyright.com. Other copying for republication, resale, advertising or promotion, or any form of systematic or multiple reproduction of any material in this book is prohibited except with permission in writing from the publisher. The CCC fee code is 0277-786X/09/\$18.00.

Printed in the United States of America.

Publication of record for individual papers is online in the SPIE Digital Library.

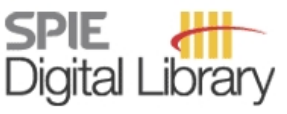

SPIEDigitalLibrary.org

Paper Numbering: Proceedings of SPIE follow an e-First publication model, with papers published first online and then in print and on CD-ROM. Papers are published as they are submitted and meet publication criteria. A unique, consistent, permanent citation identifier (CID) number is assigned to each article at the time of the first publication. Utilization of CIDs allows articles to be fully citable as soon they are published online, and connects the same identifier to all online, print, and electronic versions of the publication. SPIE uses a six-digit CID article numbering system in which:

- The first four digits correspond to the SPIE volume number.

- The last two digits indicate publication order within the volume using a Base 36 numbering system employing both numerals and letters. These two-number sets start with 00, 01, 02, 03, 04 , $05,06,07,08,09,0 A, 0 B \ldots$. OZ, followed by 10-1Z, 20-2Z, etc.

The CID number appears on each page of the manuscript. The complete citation is used on the first page, and an abbreviated version on subsequent pages. Numbers in the index correspond to the last two digits of the six-digit CID number. 


\section{Contents}

vii Conference Committee

\section{SESSION 1 SAPPHIRE AND ALUMINA}

730202 Evolution of the sapphire industry: Rubicon Technology and Gavish [7302-01]

D. C. Harris, Naval Air Systems Command (United States)

730203 Characteristics of thick (>12 mm) Class ${ }^{225}$ EFG sapphire sheet for IR window applications [7302-02]

J. Locher, C. Jones, H. Bates, J. Rioux, Saint-Gobain Crystals (United States)

730204 Hydrogen effect on the properties of sapphire [7302-03]

R. N. Mogilevsky, L. G. Sharafutdinova, Emerging Material Technologies, Inc. (United States);

S. Nedilko, Kyiv National Taras Shevchenko Univ. (Ukraine); V. Gavrilov, JSC Technocrystal (Ukraine); D. Verbilo, Frantsevich Institute for Problems of Materials Science (Ukraine);

S. D. Mittl, Insaco, Inc. (United States)

730205 Polycrystalline alumina for aerodynamic IR domes and windows [7302-04]

M. V. Parish, M. R. Pascucci, CeraNova Corp. (United States)

\section{SESSION 2 MULTIFUNCTIONAL WINDOWS AND DOMES: EVOLVING OPTICAL CHALLENGES}

730206 Large-area ALON windows for reconnaissance and armor applications [7302-06]

L. M. Goldman, R. Twedt, R. Foti, M. Smith, S. A. Sastri, Surmet Corp. (United States)

730207 Dual IR/RF windows for laser communications [7302-07]

L. M. Goldman, R. Twedt, J. Zigman, Surmet Corp. (United States); R. Ondercin, Air Force Research Lab. (United States)

730208 Joining transparent ceramics [7302-08]

A. Shechter, B. Bloch, E. Galun, Elbit Systems Electro-Optics El-Op Ltd. (Israel)

730209 Processing method and process modeling of large aperture transparent magnesium aluminate spinel domes [7302-09]

J. Yu, B. McWilliams, S. Kilczewski, G. Gilde, A. Lidie, J. Sands, U.S. Army Research Lab. (United States)

7302 OA Bonding force determination at AFB interfaces of single crystal sapphire composites [7302-10]

H.-C. Lee, H. Meissner, X. Mu, Onyx Optics, Inc. (United States); C. Liu, W. Qiu, Valdosta Optics Lab., Inc. (United States) 
7302 OB Evaluation of commercially available bulk $\mathbf{M g}$ and $\mathrm{Al}$ oxides and hydroxides for the production of transparent $\mathrm{MgAl}_{2} \mathrm{O}_{4}$ [7302-1 1]

A. C. Sutorik, G. Gilde, S. M. Kilczewski, A. Lidie, U.S. Army Research Lab. (United States)

7302 OC Observations during the fabrication of spinel optics [7302-12]

J. R. Bashe, D. L. Hibbard, Exotic Electro-Optics (United States)

7302 OD Manufacturing issues for polycrystalline transparent spinel domes [7302-13]

A. LaRoche, J. Kutsch, K. Rozenberg, L. Fehrenbacher, Technology Assessment and Transfer (United States)

7302 OE Advances in spinel ceramic technology for large windows and domes [7302-14] J. L. Sepulveda, R. O. Loutfy, S. Chang, S. Ibrahim, Materials and Electrochemical Research Corp. (United States); N. Traggis, Precision Photonics Corp. (United States)

7302 OF Recent developments in transparent polycrystalline spinel for electro-optic applications [7302-15]

I. Aggarwal, S. Bayya, G. Villalobos, W. Kim, J. Sanghera, Naval Research Lab. (United States)

7302 OG High-strength transparent spinel with fine, unimodal grain size [7302-16]

S. M. Sweeney, M. K. Brun, T. J. Yosenick, A. Kebbede, M. Manoharan, GE Global Research (United States)

\section{SESSION 4 COATINGS AND SURFACE TREATMENTS}

$7302 \mathrm{OH} \quad$ High growth rate deposition of highly oriented polycrystalline diamond film [7302-17]

C. Liu, W. Qiu, Valdosta Optics Lab., Inc. (United States); H. Meissner, Onyx Optics, Inc. (USA)

7302 ol Evaluating environmental survivability of optical coatings [7302-18]

S. Joseph, D. Yadlovker, O. Marcovitch, H. Zipin, Rafael Advanced Defense Systems Ltd. (Israel)

7302 0J Study of the environmental and optical durability of AR microstructures in sapphire, ALON, and diamond [7302-20]

D. S. Hobbs, TelAztec LLC (United States)

7302 OK Application of physical gradient index (Moth-Eye) structures to ALON windows as a durable anti-reflection treatment [7302-21]

N. Agarwal, L. M. Goldman, S. A. Sastri, Surmet Corp. (United States); R. J. Ondercin, Air Force Research Lab. (United States); P. Kobrin, Teledyne Scientific \& Imaging LLC (United States)

\section{SESSION 5 GERMANIUM AND ZINC SULFIDE OPTICAL MATERIALS}

$7302 \mathrm{OL} \quad$ A historical view of germanium as an infrared window material [7302-22]

R. M. Sullivan, Office of Naval Research (United States) 
$73020 \mathrm{M}$ Variability in chemical vapor deposited zinc sulfide: assessment of legacy and international CVD ZnS materials [7302-24]

J. McCloy, Raytheon Missle Systems (United States); R. Korenstein, Raytheon Co. (United States)

$7302 \mathrm{ON}$ The effect of metal on the formation of multispectral zinc sulfide [7302-25]

J. McCloy, Raytheon Missile Systems (United States); R. Korenstein, Raytheon Co. (United States)

730200 Varying electro-kinetic interactions to achieve predictable polishing results on zinc sulfide [7302-26]

J. DeGroote Nelson, J. A. Drucker, A. A. Haefner, R. A. Wiederhold, Optimax Systems, Inc. (United States)

\section{SESSION 6 METROLOGY}

7302 OP Instrumentation for characterizing a new class of optical domes [7302-27]

A. K. Lal, J. S. Jo, S. Kupiec, E. Scott, J. Trolinger, MetroLaser, Inc. (United States)

7302 OQ Round robin testing of the Optimax Optidome [7302-28]

M. Martucci, R. Plympton, Optimax Systems, Inc. (United States)

7302 OR Measurement results for time-delayed source interferometers for windows, hemispherical domes, and tangent ogives [7302-29]

W. P. Kuhn, Opt-E (United States); M. B. Dubin, College of Optical Sciences, The Univ. of Arizona (United States); R. S. LeCompte, H. P. Durazo, Breault Research Organization, Inc. (United States)

7302 OS An innovative non-contact surface measurement solution for asphere, deep parabolic, and ogive radome geometries [7302-30]

S. DeFisher, M. Bechtold, D. Mohring, S. Bambrick, OptiPro Systems (United States)

7302 OT Low-coherence surface metrology using a multiple-beam optical probe [7302-31]

D. W. Diehl, C. J. Ditchman, C. T. Cotton, ASE Optics, Inc. (United States)

\section{SESSION 7 CHARACTERIZATION AND FINISHING}

7302 OU Recent developments in finishing of deep concave, aspheric, and plano surfaces utilizing the Ultraform 5-axes computer controlled system [7302-33]

S. Bambrick, M. Bechtold, S. DeFisher, D. Mohring, J. Meisenzahl, OptiPro Systems (United States)

7302 OV Technologies for precision manufacture of current and future windows and domes [7302-34] B. Hallock, A. Shorey, QED Technologies (United States)

7302 OW Semi-empirical scattering model for chemical vapor deposited zinc sulfide [7302-35] J. McCloy, Raytheon Missile Systems (United States) 
7302 OX Predicted and measured EMI shielding effectiveness of a metallic mesh coating on a sapphire window over a broad frequency range [7302-36]

K. T. Jacoby, M. W. Pieratt, Exotic Electro-Optics, Inc. (United States); J. I. Halman,

K. A. Ramsey, Battelle Memorial Institute (United States)

7302 OY Predicted and measured transmission and diffraction by a metallic mesh coating [7302-37] J. I. Halman, K. A. Ramsey, Battelle Memorial Institute (United States); M. Thomas, A. Griffin, Spica Technologies, Inc. (United States)

$7302 \mathrm{OZ} \quad$ Birefringence and grain-size effects on optical transmittance of polycrystalline magnesium fluoride [7302-38]

T.-C. Wen, D. K. Shetty, The Univ. of Utah (United States)

$730210 \quad$ Flexural strength of fused silica: Weibull statistical analysis [7302-39]

C. A. Klein, C.A.K. Analytics, Inc. (United States)

SESSION 8 NOVEL MATERIALS

730211 Low-thermal expansion infrared glass ceramics [7302-40]

P. Lam, L2 Tech, Inc. (United States)

730212 Optical properties of polycrystalline Nd:YAG for different levels of Nd doping [7302-42] R. M. Springer, Naval Air Systems Command (United States); M. E. Thomas, D. V. Hahn, The Johns Hopkins Univ. Applied Physics Lab. (United States); F. A. Narducci, Naval Air Systems Command (United States)

730213 Onshore produced ceramic laser material development progress [7302-43] J. C. Huie Imholt, T. Hartnett, R. Gentilman, S. R. Silberstein, Raytheon Integrated Defense Systems (United States)

730214 Advanced parylene technology for sensors, optical windows, and display applications [7302-44]

R. Kumar, Specialty Coating Systems, Inc. (United States)

\section{POSTER SESSION}

730215 Design and implementation of a new time-delayed source and alignment considerations for a tangent ogive interferometer [7302-45]

H. P. Durazo, Breault Research Organization (United States); W. P. Kuhn, Opt-E (United States); R. S. LeCompte, Breault Research Organization (United States); M. B. Dubin, College of Optical Sciences, The Univ. of Arizona (United States)

Author Index 


\title{
Conference Committee
}

\author{
Symposium Chair \\ Ray O. Johnson, Lockheed Martin Corporation (United States) \\ Symposium Cochair
}

Michael T. Eismann, Air Force Research Laboratory (United States)

Conference Chair

Randal W. Tustison, Raytheon Co. (United States)

Program Committee

Ishwar D. Aggarwal, Naval Research Laboratory (United States)

Joel Askinazi, Goodrich Corporation (United States)

Richard Gentilman, Raytheon Company (United States)

Daniel C. Harris, Naval Air Systems Command (United States)

Brian K. Jones, U.S. Army Research, Development and Engineering Command (United States)

James C. Kirsch, U.S. Army Research, Development and Engineering Command (United States)

Robert J. Ondercin, Air Force Research Laboratory (United States)

Adrienne E. Selz, Air Force Research Laboratory (United States)

Roger M. Sullivan, Office of Naval Research (United States)

Michael E. Thomas, The Johns Hopkins University Applied Physics Laboratory (United States)

Brian J. Zelinski, Raytheon Missile Systems (United States)

\section{Session Chairs}

1 Sapphire and Alumina

Daniel C. Harris, Naval Air Systems Command (United States)

2 Multifunctional Windows and Domes: Evolving Optical Challenges

Robert J. Ondercin, Air Force Research Laboratory (United States)

3 Spinel Windows and Domes

Joel Askinazi, Goodrich Corporation (United States)

$4 \quad$ Coatings and Surface Treatments

Richard Gentilman, Raytheon Company (United States) 
$5 \quad$ Germanium and Zinc Sulfide Optical Materials

Adrienne E. Selz, Air Force Research Laboratory (United States)

6 Metrology

Ishwar D. Aggarwal, Naval Research Laboratory (United States)

$7 \quad$ Characterization and Finishing

Roger M. Sullivan, Office of Naval Research (United States)

8 Novel Materials

Michael E. Thomas, The Johns Hopkins University Applied Physics Laboratory (United States) 